\title{
The Relationship Between Morning Symptoms and the Risk of Future Exacerbations in COPD
}

This article was published in the following Dove Press journal:

International Journal of Chronic Obstructive Pulmonary Disease

\author{
Tian Sun ${ }^{1,2}$ \\ Xiaoyun $\mathrm{Li}^{3}$ \\ Wei Cheng (D) ${ }^{1,2}$ \\ Yating Peng $\mathbb{D}^{1,2}$ \\ Yiyang Zhao (iD) ${ }^{1,2}$ \\ Cong Liu ${ }^{1,2}$ \\ Yuqin Zeng ${ }^{1,2}$ \\ Yan Chen ${ }^{1,2}$ \\ Shan $\mathrm{Cai}^{1,2}$ \\ Ping Chen $\mathbb{D}^{1,2}$
}

'Department of Pulmonary and Critical Care Medicine, The Second Xiangya Hospital, Central South University, Changsha, Hunan 4100II, People's Republic of China; ${ }^{2}$ Research Unit of Respiratory Disease, Central South University, Central South University, Changsha, Hunan 4100II, People's Republic of China; ${ }^{3}$ Department of Respiratory and Critical Care Medicine, The First Affiliated Hospital, Sun Yat-Sen University, Guangzhou, Guangdong 510000, People's Republic of China
Correspondence: Ping Chen Department of Pulmonary and Critical Care Medicine, The Second Xiangya Hospital I39

Renmin Middle Road, Changsha, Hunan

4100 I I, People's Republic of China

Tel +86- | 3873 I I5563

Fax $+86731-85295848$

Email pingchen0731@csu.edu.cn
Background: The morning is the most troublesome time of day for patients with chronic obstructive pulmonary disease (COPD). However, the association of morning symptoms and COPD exacerbations in longitudinal follow-up has not been studied. In this study, we mainly aimed to investigate the relationship between morning symptoms and exacerbations over a one-year follow-up period. And the secondary aim was an investigation of the association between morning symptoms and baseline clinical features.

Patients and Methods: Ninety-two patients with stable COPD provided the baseline information. Morning symptoms were assessed with the Chinese version of Chronic Obstructive Pulmonary Disease Morning Symptom Diary (Ch-COPD-MSD); the median morning symptoms score was used as a cut-off to separate the study cohort in two groups. Modified Medical Research Council (mMRC), COPD assessment test (CAT), and Clinical COPD Questionnaire (CCQ) were used and exacerbation history of the previous year was recorded. Seventy-eight patients $(84.8 \%)$ completed the longitudinal follow-up of exacerbations.

Results: The median morning symptoms score was 30 in stable COPD patients. Morning symptoms severity was different between COPD Global Initiative for Chronic Obstructive Lung Disease (GOLD) groups $(p<0.001)$. Patients with high morning symptoms (score $>30$ ) had higher scores of mMRC, CAT, and CCQ $(p<0.05)$. CAT score was an independent risk factor of morning symptoms. During follow-up, $41 \%$ of patients experienced $\geq 1$ exacerbation. The frequency of severe exacerbations was higher in patients with high morning symptoms compared to patients with low morning symptoms $(p<0.005)$. The Ch-COPDMSD score could predict future severe exacerbations; the area under the ROC curve was 0.751 (95\% CI: $0.633-0.868, p=0.002$ ).

Conclusion: Worse health status and more dyspnea symptom were associated with increased severity of morning symptoms. Morning symptoms were most strongly related to future severe exacerbations and could predict future exacerbations in patients with COPD.

Keywords: chronic obstructive pulmonary disease, morning symptoms, future exacerbations

\section{Introduction}

Chronic obstructive pulmonary disease (COPD) is a common respiratory disease, serious harm to human health. ${ }^{1}$ With the prevalence increasing, the World Health Organization (WHO) revealed that COPD would become the third leading cause of death worldwide by $2030 .^{2}$ COPD clinical manifestations are diverse, and its main presentation includes dyspnea, chronic cough and expectoration, breathing chest tightness, and other characteristics (such as fatigue, weight loss, anorexia, etc.). ${ }^{1}$ Studies have shown that the morning is the worst time. Morning symptoms of patients with COPD produce a certain effect on the patient's health status and daily life ability. ${ }^{3-7}$ 
Morning symptoms variability correlated with older patients who were more active smokers with worse lung function and poorer health status, greater COPD duration, greater incidence of exacerbation in the past 12 months, higher levels of anxiety/depression, and poor medication adherence than those without morning symptoms variability. ${ }^{3,5,6,8-11}$ Some scholars have developed a quantitative assessment scale for tools used in the evaluation of the COPD morning symptoms, such as chronic obstructive pulmonary disease morning symptom diary (COPD-MSD), ${ }^{12}$ PRO-morning symptoms questionnaire, ${ }^{13}$ morning daily life ability questionnaire (CDLM questionnaire), and integrated chest symptoms questionnaire (GCSQ questionnaire), ${ }^{14}$ and others. Previous research shows that the Chinese version of COPD-MSD (ChCOPD-MSD) has good reliability and validity, which can help clinicians to assess patients with COPD morning symptoms in China. ${ }^{15}$

Exacerbations of COPD directly affect the course of the disease, which is associated with a faster rate of lung function decline, ${ }^{16}$ impaired health status, ${ }^{16,17}$ and increased risk of mortality, ${ }^{18,19}$ and is a vital indicator evaluation of the longterm risk of poor prognosis. ${ }^{20}$ Thus, reducing the risk of exacerbations is a crucial goal of COPD therapy. Existing studies have shown that COPD morning symptoms and exacerbations are correlated. ${ }^{10,21}$ The rate and severity of COPD exacerbations were associated with the severity of airflow limitation, but the history of exacerbations was the best predictor of future exacerbations. ${ }^{22}$ Other factors independently associated with an increased risk of exacerbations include female gender, poor lung function, poor health status, low level of physical activity, anxiety, and depression. ${ }^{23-27}$ To date, no studies have definitively shown that COPD morning symptoms were associated with a risk of future exacerbations in COPD.

In the study, the main purpose was an investigation of the relationship between morning symptoms and future risk of exacerbation in COPD patients. Moreover, the secondary purpose was an investigation of the association between morning symptoms and baseline clinical features.

\section{Patients and Methods Study Design}

A cross-sectional and longitudinal study with outpatient subjects was carried out in the Department of Respiratory and Critical Care Medicine, at the Second Xiangya Hospital, Central South University, Changsha, Hunan, China, from June 24, 2018, to October 31, 2018.
A researcher explained the purpose of the study at enrollment and written informed consent was obtained from all study participants. The study was approved by the institutional review board of the Second Xiangya Hospital of Central South University (Hunan, China) and conducted according to the Declaration of Helsinki (Registration number: ChiCTR-POC-17,010,431).

\section{Study Populations}

Enrolled patients were diagnosed with COPD (defined by the 2017 GOLD recommendations: spirometry with a ratio of the forced expiratory volume in $1 \mathrm{~s}$ to the forced vital capacity (FEV1/FVC) lower than 0.70 after bronchodilator administration), ${ }^{1}$ and were aged 40 to 75 years. They had at least one symptom of COPD (chronic cough, sputum, dyspnea, chest tightness, wheezing, etc.). Besides, they had no mental disorders that limited their ability to provide informed consent, complete the questionnaires, and finish spirometry. Exclusion criteria were patients with other chronic respiratory diseases, such as bronchiectasis, asthma, interstitial lung disease, tuberculosis, and lung cancer. Patients were excluded if they had clinically significant unstable/uncontrolled heart, liver, kidney, and other systemic diseases. Patients with a history of mental illness and severe mental retardation unable to communicate were also excluded as were patients with an exacerbation in the previous four weeks. Thus, 108 patients were enrolled in the study. Sixteen patients were excluded because they no longer met the study inclusion criteria, so 92 patient completed baseline data collection. In the 1-year follow-up period, 14 patients dropped out of the study, and two of them died for reasons not related to COPD exacerbations. Thus, 78 patients had satisfactory outcomes for analyses (Figure 1).

\section{Study Procedures and Outcomes}

Patients had a baseline visit (Day 1) and a follow-up interview after one year. Baseline information was collected on Day 1 including age, sex, height, weight, education, and smoking status, some questionnaires, COPD exacerbations during the previous year. During the oneyear follow-up interview, physicians collected COPD exacerbations during the period since baseline by checking medical records and interviewing patients. Patients completed all of the self-report questionnaires by paper.

The occurrence and severity of morning symptoms were assessed with the Ch-COPD-MSD: 19 items total with the total value ranged from 19 to $101 .{ }^{12,15}$ The 


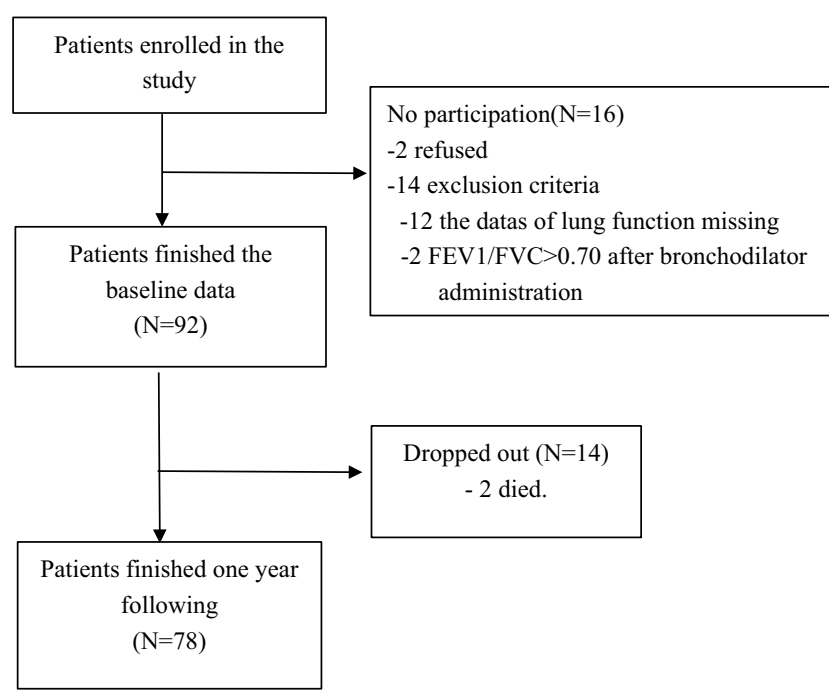

Figure I Study flow diagram

median morning symptoms score was used to create two groups, patients with high morning symptoms (the morning symptoms score $\geq 30$ ), and patients with low morning symptoms (the morning symptoms score $<30$ ). Morning symptoms were defined as those present when getting up in the morning. ${ }^{10}$

Also, dyspnea was measured by using mMRC. ${ }^{1}$ The CAT and the CCQ were used to assess health status. ${ }^{28}$ The GOLD $\mathrm{ABCD}$ group was classified based on the patient's symptoms and exacerbation history in the last 12 months. ${ }^{1}$ COPD disease severity was classified using the GOLD guidelines and was divided into four stages: mild (stage I), moderate (stage II), severe (stage III), or very severe (stage IV). ${ }^{1}$

A COPD exacerbation was defined as an acute event characterized by a worsening of the patient's respiratory symptoms that were beyond day-to-day variations and led to a change in medication. According to the 2017 GOLD document, ${ }^{1}$ COPD exacerbations are classified as mild (treated with short-acting bronchodilators (SABDs) only), moderate (treated with SABDs plus antibiotics and/or oral corticosteroids), severe (patient requires hospitalization or visits the emergency room). And frequent exacerbations were defined as two or more exacerbations per year. ${ }^{1}$ In this study, the exacerbations were recorded according to the criteria of moderate/severe exacerbations.

\section{Statistical Analyses}

Statistical analyses were performed using SPSS version 25.0. Descriptive data were reported as percentages, mean values standard deviations (SD) for normally distributed continuous variables and median with interquartile ranges (IQR) for non-normal continuous variables. Differences in baseline characteristics between the two groups were compared with an independent $t$-test for continuous normally distributed variables; a Mann-Whitney $U$-test for continuous non-normal distributed variables and $\chi^{2}$ test for categorical variables. The Kruskal-Wallis $H$-test was used for multiple-group comparisons. The association between various parameters and morning symptom severity was analyzed with Spearman's rank correlation coefficient and multiple linear regression analysis. The ROC curve was drawn, and the area under the curve (AUC) was compared by Z-test. For all analyses, a $p$-value of $<0.05$ was considered statistically significant.

\section{Results}

\section{Patients}

Table 1 shows the demographics and baseline characteristics of 92 stable COPD patients. Patients were (mean \pm SD) $58.6 \pm 7.7$ years old, and $85.9 \%$ were male. Most patients were associated with smoking, either current (34.8\%) or former $(54.3 \%)$ smokers. They had a mean FEV1 of $52.7 \pm 21 \%$ predicted. Most patients were classified as COPD GOLD B (38.0\%) and D (44.6\%), also GOLD II (45.7\%) and III (30.4\%). As high as $55 \%$ of patients had a history of exacerbation in the past year.

\section{Characteristics of Morning Symptoms}

Morning symptoms score ranged from 19 to 81 . The median morning symptoms score was 30.0 and was used as a cut-off to separate the study cohort in two groups (Table 1). Morning symptoms severity was no different between COPD stages: median (IQR) score in GOLD I was 25.5 (22.5-37.5), in GOLD II 30.0 (23.0-38.5), in GOLD III 29.0 (25.5-37.0), and in GOLD IV 43.5 (28.7553.0). Morning symptoms severity was different between COPD GOLD groups: median (IQR) score in GOLD A was 22.5 (19.0-26.5), in GOLD B 31.0 (25.0-37.0), in GOLD C 26.5 (23.25-33.5), and in GOLD D 37.0 (27.049.0) $(p<0.001)$. Post hoc analyses showed differences between groups A and B ( $\mathrm{p}=0.008)$, A and D were statistically significant $(p=0.000)$ (Figure 2$)$; the difference between other groups was not statistically significant.

\section{Association of Morning Symptoms and Clinical Characteristics}

The median (IQR) score of mMRC in patients with high morning symptoms was $2(1,2)$, while in patients with low 
Table I Baseline Characteristics

\begin{tabular}{|c|c|c|c|c|}
\hline Characteristics & $\begin{array}{l}\text { All Included } \\
\text { Patients }(\mathrm{N}=92)\end{array}$ & $\begin{array}{l}\text { Morning Symptoms } \\
\text { Score }<30.0(N=45)\end{array}$ & $\begin{array}{l}\text { Morning Symptoms } \\
\text { Score } \geq 30.0(N=47)\end{array}$ & $\begin{array}{l}\text { Difference } \\
\text { (P-value) }\end{array}$ \\
\hline Age in years, mean (SD) & $58.6(7.7)$ & $57.4(7.1)$ & $59.9(8.2)$ & 0.126 \\
\hline Male, n (\%) & 79(85.9) & $40(88.9)$ & $39(83)$ & 0.416 \\
\hline Education status, n (\%) & & & & 0.262 \\
\hline High school or less & $85(92.4)$ & $40(89.9)$ & $45(95.7)$ & \\
\hline some college and above & $7(7.6)$ & $5(11.1)$ & $2(4.3)$ & \\
\hline Smoking status, n (\%) & & & & $0.44 I$ \\
\hline Current smoker & $32(34.8)$ & $16(35.6)$ & $16(34.0)$ & \\
\hline Former smoker & $50(54.3)$ & $26(57.8)$ & $24(5 I .1)$ & \\
\hline never smoked & $10(10.9)$ & $3(6.7)$ & $7(14.9)$ & \\
\hline BMI in kg/m2, mean (SD) & $22.3(3.5)$ & $22.9(3.2)$ & $21.8(3.6)$ & 0.147 \\
\hline FEVI\% predicted, mean (SD) & $52.7(21.0)$ & $55.3(20.5)$ & $50.3(21.5)$ & 0.25 \\
\hline GOLD stage, n (\%) & & & & 0.187 \\
\hline I & $10(10.9)$ & $7(15.6)$ & $3(6.4)$ & \\
\hline II & $42(45.7)$ & $20(44.4)$ & $22(46.8)$ & \\
\hline III & $28(30.4)$ & $15(33.3)$ & $13(27.7)$ & \\
\hline IV & $12(13.0)$ & $3(6.7)$ & $9(19.1)$ & \\
\hline GOLD group, n (\%) & & & & 0.001 \\
\hline$A$ & $12(13.0)$ & $12(26.7)$ & 0 & \\
\hline B & $35(38.0)$ & $14(3||)$. & $21(44.7)$ & \\
\hline C & $4(4.3)$ & $3(6.7)$ & $I(2 . I)$ & \\
\hline $\mathrm{D}$ & $4 I(44.6)$ & $16(35.6)$ & $25(53.2)$ & \\
\hline mMRC total score, median(IQR) & $2(1,2)$ & $2(1,2)$ & $2(2,3)$ & 0.002 \\
\hline CAT total score, mean (SD) & I4.4(6.6) & $10.6(5.6)$ & $18.1(5.3)$ & 0.000 \\
\hline CCQ total score, mean (SD) & $2.0(0.7)$ & $1.7(0.6)$ & $2.3(0.6)$ & 0.000 \\
\hline Exacerbations in the previous year, $n(\%)$ & $55(59.8)$ & $25(55.6)$ & $30(63.8)$ & 0.418 \\
\hline
\end{tabular}

Notes: Data are presented as the mean (SD), median (IQR), or number (\%). Data were compared between two groups using Mann-Whitney $U$-test, $t$-test, and $2 \chi$-test. Abbreviations: FEVI\% predicted, forced expiratory volume in one second as a percentage of the predicted value; BMI, body mass index; mMRC, Modified Medical Research Council Dyspnea Scale; CAT, COPD assessment test; CCQ, Clinical COPD questionnaire; GOLD, Global Initiative for Chronic Obstructive Lung Disease; SD, standard deviation; IQR, interquartile ranges.

morning symptoms it was $2(2,3)$, which was significantly different ( $p=0.002$; Figure 3A). Patients with high morning symptoms presented a significantly higher CAT total score compared to those with low morning symptoms (18.1 vs 16.0, $p=0.000$; Figure 3B). Additionally, the CCQ total score was significantly higher in patients with high morning symptoms compared to those with low morning symptoms ( 2.3 vs $1.7, p=0.000$; Figure $3 \mathrm{C}$ ).

\section{Correlations Between Morning}

\section{Symptoms and Clinical Characteristics}

Correlations between clinical characteristics of COPD patients and morning symptoms were evaluated using Spearman's rank correlation coefficient. FEV1\% predicted was significantly correlated with morning symptoms $(\mathrm{r}=-0.22, p<0.05$;
Figure 4A). Scores of mMRC, CAT and CCQ were significantly correlated with morning symptoms $(\mathrm{r}=0.41, p<0.001$; $\mathrm{r}=0.67, p<0.001 ; \mathrm{r}=0.48, p<0.001$; Figure 4B-D). Multiple linear regression analysis was performed to test the associations of morning symptoms severity with other variables, including age, sex, FEV1\% predicted, mMRC score, CAT score, CCQ score, and exacerbations in the past year. Table 2 shows the health status (CAT score) was associated with morning symptoms severity $(\mathrm{B}=1.255, p=0.000)$.

\section{Relationship Between Morning Symptoms and Exacerbations Over One Year}

During the one-year follow-up period, $41 \%$ of patients experienced at least one-time exacerbations. The number of severe exacerbations was higher in patients with high 


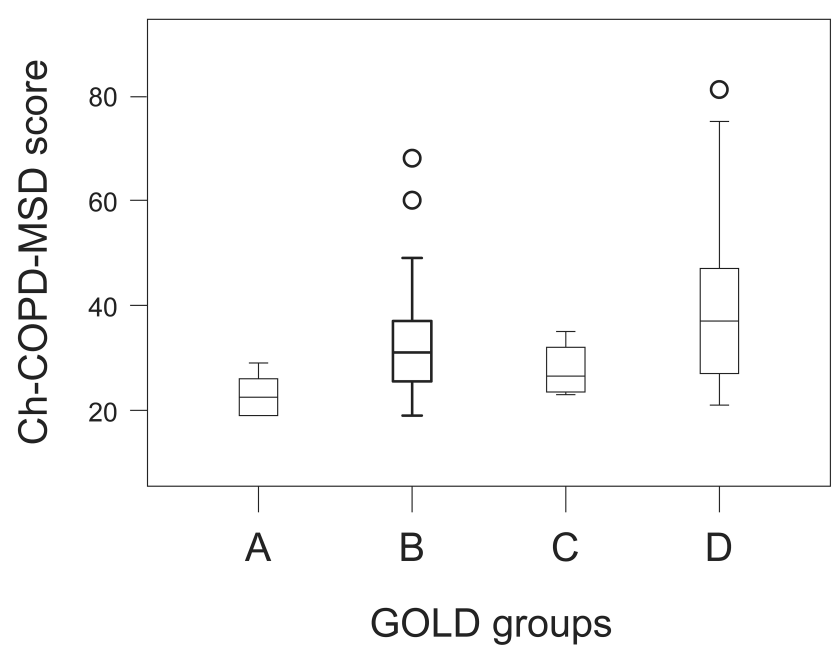

Figure 2 Morning symptom score in COPD GOLD A, B, C and D groups. COPD GOLD A $(n=12), B(n=35), C(n=4)$ and $D(n=41)$. Data were compared between groups using the Kruskal-Wallis $H$-test.

Abbreviations: GOLD, Global Initiative for Chronic Obstructive Lung Disease; Ch-COPD-MSD, Chinese-version of chronic obstructive pulmonary disease morning symptom diary.

morning symptoms compared with patients who had low morning symptoms. Meanwhile, the number of patients with severe exacerbations was higher in patients with high morning symptoms (35.5\%) compared with patients who had low morning symptoms (7.7\%) (all $p<0.005$, Table 3 ).

Figure 5 shows the ROC curve of severe exacerbations of COPD patients at a stable period was predicted by $\mathrm{Ch}$ COPD-MSD score. The area under the ROC curve was 0.751 (95\% CI: $0.633-0.868, p=0.002$ ).

\section{Discussion}

To our knowledge, this cross-sectional and longitudinal study is the first to explore the relationship between morning symptoms and the risk of future exacerbations. We found a significant correlation of the severity of morning symptoms with the risk of future severe exacerbations. Moreover, the severity of morning symptoms predicted the risk of future severe exacerbations in stable COPD patients and was associated with health status.

Chronic obstructive pulmonary disease morning symptom diary (COPD-MSD) was developed for quantitative evaluation of morning symptoms in patients with COPD in 2016. ${ }^{12}$ Soon after, the Chinese-version of chronic obstructive pulmonary disease morning symptom diary (Ch-COPDMSD) was developed for clinicians to assess patients with COPD morning symptoms in China. ${ }^{15}$ Although there is no consistent morning symptom questionnaire available yet, we think that the Ch-COPD-MSD can be a suitable tool to assess morning symptoms. As shown, ${ }^{29}$ morning symptoms were evaluated with the PRO-morning COPD Symptoms Questionnaire, and the median score was used to create two groups (low and high morning symptom scores). Thus, the median morning symptoms score of the Ch-COPD-MSD was used as a cut-off to separate the study cohort in two groups in our study. A validated cut-off score might help to determine whether the median score of 30.0 could be seen as high or as low impact.

We did not observe significant differences in age, sex, smoking status, education status, BMI, and lung function with patients having different morning symptoms scores, which ensured the comparability of basic characteristics between the two groups. This result was consistent with the results of a multi-center study conducted by Peking University Third Hospital. ${ }^{17}$ Morning symptoms score was higher in patients with GOLD $\mathrm{B}$ and GOLD D compared with GOLD-A and GOLD $\mathrm{C}$ consistent with the results of previous studies ${ }^{11}$ Using
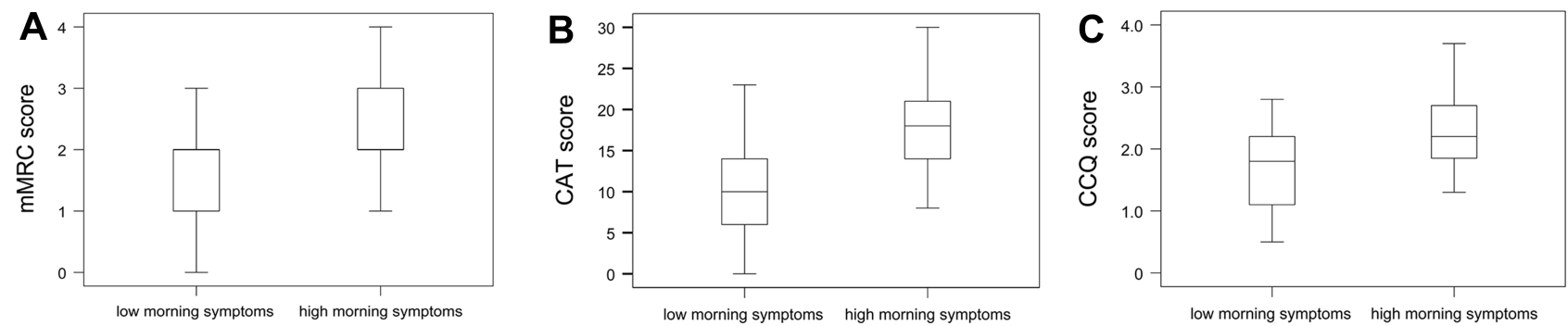

Figure 3 Association of morning symptom and clinical characteristics in COPD patients. (A) Comparison of baseline mMRC score between the two groups. (B) Comparison of baseline CAT score between the two groups. (C) Comparison of baseline CCQ score between the two groups. Data were compared between groups using the Mann-Whitney $U$-test and $t$-test.

Abbreviations: mMRC, Modified Medical Research Council Dyspnea Scale; CAT, COPD assessment test; CCQ, Clinical COPD questionnaire. 

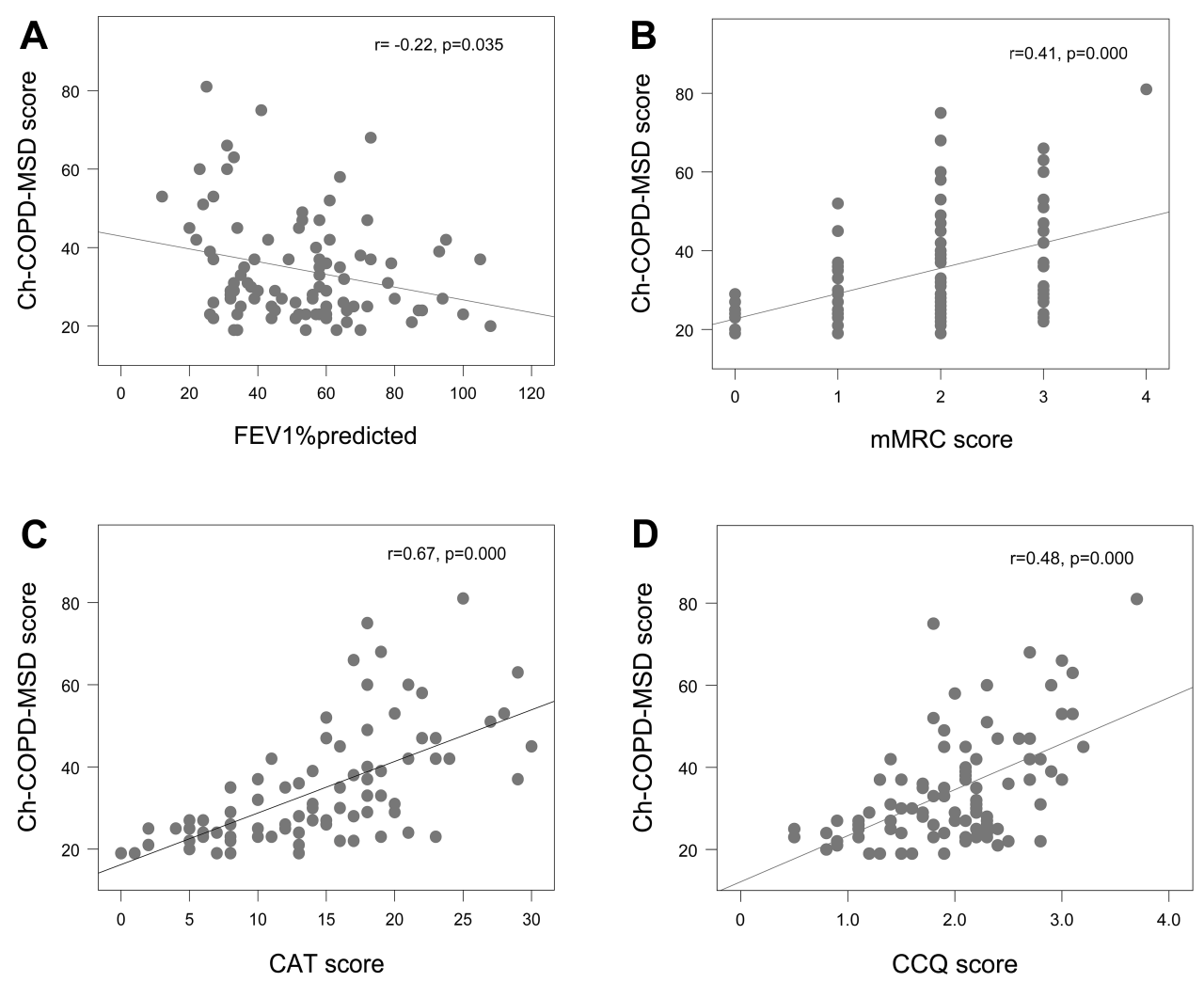

Figure 4 Correlation between morning symptom and clinical characteristics. (A) Correlation between Ch-COPD-MSD score and FEVI\% predicted in COPD patients. (B) Correlation between Ch-COPD-MSD score and mMRC score in COPD patients. (C) Correlation between Ch-COPD-MSD score and CAT score in COPD patients. (D) Correlation between ChCOPD-MSD score and CCQ score in COPD patients. Correlations between continuous variables were evaluated using Spearman's rank correlation coefficient.

Abbreviations: FEVI\% predicted, forced expiratory volume in one second as a percentage of the predicted value; mMRC, Modified Medical Research Council Dyspnea Scale; CAT, COPD assessment test; CCQ, Clinical COPD questionnaire; Ch-COPD-MSD, Chinese-version of chronic obstructive pulmonary disease morning symptom diary.

single-factor analysis, we found that patients with high morning symptoms had higher mMRC, CAT and CCQ scores. This result was consistent with the conclusion of previous multi-factor analysis. ${ }^{10,30}$

In this study, the morning symptoms were negatively correlated with lung function, while positively - with mMRC, CAT, and CCQ score. In the multiple linear regression analysis, we found that poorer health was associated with more severe morning symptoms, a finding confirmed by previous studies in which CAT score was an independent risk factor for morning symptoms. ${ }^{10,17}$

During the follow-up, $41 \%$ of patients experienced at least one time exacerbations. The number of severe exacerbations was higher in patients with high morning symptoms compared with patients who had low morning symptoms. Meanwhile, the number of patients with severe exacerbations was higher in patients with high morning symptoms (35.5\%) compared with patients who had low

Table 2 Associations Between Clinical Characteristics and Morning Symptoms Severity According to Multiple Linear Regression Analysis

\begin{tabular}{|l|l|l|l|l|l|}
\hline & \multicolumn{2}{|l|}{ Unstandardized Coefficients } & \multicolumn{2}{l|}{ Standardized Coefficients } & P-value \\
\cline { 2 - 6 } & B & SE & $\boldsymbol{\beta}$ & t \\
\hline Constant & 16.227 & 2.674 & & 6.069 & 0.000 \\
CAT score & 1.255 & 0.169 & 0.617 & 7.447 & 0.000 \\
\hline
\end{tabular}

Notes: age, sex, FEVI\% predicted, mMRC score, CAT score, CCQ score and exacerbations in the past year were included as the independent variables in the model. Abbreviations: FEVI\% predicted, forced expiratory volume in one second as a percentage of the predicted value; mMRC, Modified Medical Research Council Dyspnea Scale; CAT, COPD assessment test; CCQ, Clinical COPD questionnaire; SE, standard error. 
Table 3 The Relationship Between Morning Symptoms and the Exacerbations of the One-Year Follow-Up Period

\begin{tabular}{|c|c|c|c|c|}
\hline & $\begin{array}{l}\text { All Patients } \\
(\mathbf{N}=\mathbf{7 8})\end{array}$ & $\begin{array}{l}\text { Morning Symptoms Score < } \\
30.0(\mathrm{~N}=39)\end{array}$ & $\begin{array}{l}\text { Morning Symptoms Score } \geq \\
30.0(\mathrm{~N}=39)\end{array}$ & P-value \\
\hline \multicolumn{5}{|l|}{ Exacerbations during follow-up, $\mathrm{n}$ (\%) } \\
\hline No exacerbation & $46(59.0)$ & $27(69.2)$ & $19(48.7)$ & 0.066 \\
\hline Moderate exacerbations & $22(28.2)$ & $\mathrm{II}(28.2)$ & $I I(28.2)$ & 1.000 \\
\hline Severe exacerbations & $\mid 7(2 \mid .8)$ & $3(7.7)$ & I4(35.9) & 0.003 \\
\hline Frequent exacerbations & $17(21.8)$ & $6(15.4)$ & $\mathrm{II}(28.2)$ & 0.170 \\
\hline \multicolumn{5}{|l|}{$\begin{array}{l}\text { Exacerbations during follow- } \\
\text { up, median [IQR] }\end{array}$} \\
\hline Total exacerbations & $0(0, I)$ & $0(0, I)$ & $I(0,2)$ & 0.950 \\
\hline Moderate exacerbations & $0(0, I)$ & $0(0,1)$ & $0(0, I)$ & 0.002 \\
\hline Severe exacerbations & $0(0,0)$ & $0(0,0)$ & $0(0,1)$ & 0.078 \\
\hline
\end{tabular}

Notes: Data are presented as the median (IQR), or number (\%). Data were compared between two groups using Mann-Whitney $U$-test, and $2 \chi$-test. Abbreviations: SD, standard deviation; IQR, interquartile ranges.

morning symptoms (7.7\%) (all $p<0.005)$. Previous followup studies showed that patients with morning symptoms were more likely to have at least one exacerbation during the follow-up period of the next 6 months; those with daytime and nighttime symptoms were more likely to have a future exacerbation. ${ }^{6}$ Also, in a separate study, morning symptoms were the main predictor of future deterioration compared to nighttime symptoms when the

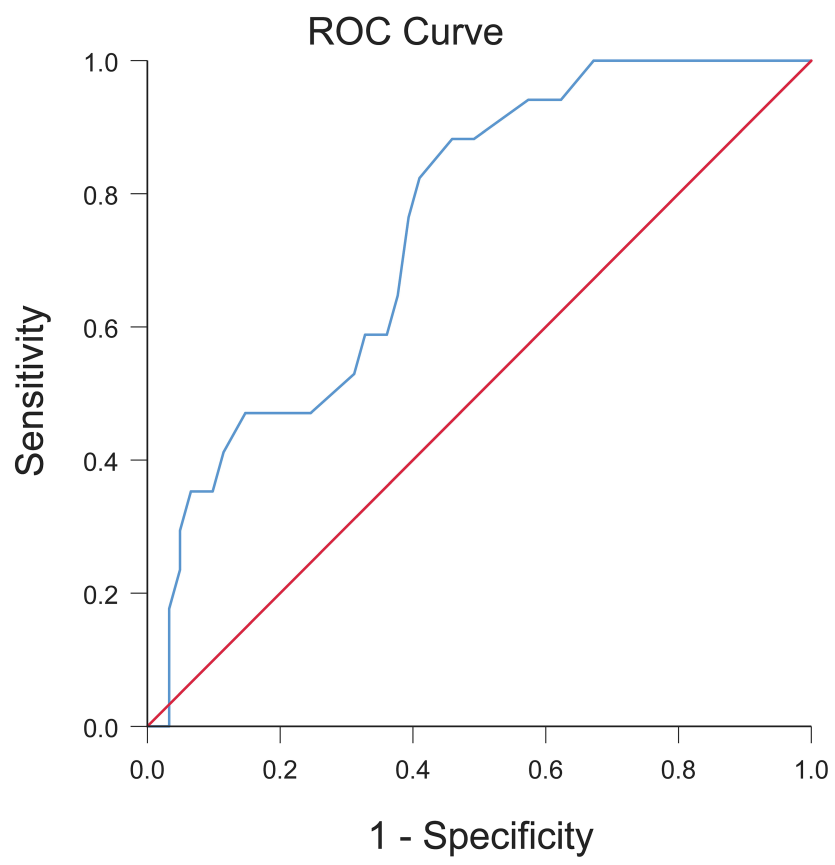

Figure $\mathbf{5}$ The ROC curve of severe exacerbation of COPD patients was predicted by Ch-COPD-MSD score.

Abbreviations: Ch-COPD-MSD, Chinese-version of chronic obstructive pulmonary disease morning symptom diary; ROC, receiver operating characteristic. risk of future exacerbations was assessed by including both nighttime symptoms and morning symptoms. ${ }^{11}$

In this study, severe exacerbations were defined as hospitalized or emergency patients. Therefore, it can be speculated that morning symptoms are related to the risk of future hospitalization. This study further analyzed whether morning symptoms could predict the risk of severe exacerbations of COPD, and found that the area under the ROC curve was 0.751 (95\% CI: $0.633-0.868$, $p=0.002$ ). This finding is the breakthrough point of this paper, which will help to indirectly assess the risk of severe exacerbations in the future patients with morning symptoms. In the current study, we cannot answer why patients with morning symptoms experience more exacerbations. It may be related to morning symptoms affecting their physical activities, health status, even medication adherence. Thus, more objective research should focus on morning symptoms, activity limitations, and medication adherence of COPD patients.

There were some limitations to the study. Firstly, this study was limited to one center, so multi-center large samples study is needed in future. Secondly, more male than female patients were enrolled in this study, while increased exacerbation risk is associated with the female gender. Also, the study is not generalizable to the global COPD population since the study was only performed in Chinese subjects. Furthermore, we did not control for comorbidities and medications.

\section{Conclusion}

Patients with high morning symptoms score were associated with severe exacerbations in COPD stable patients 
in future. A score of the Ch-COPD-MSD could predict future exacerbation in patients with COPD. Also, patients with the higher morning symptoms score had worse health status.

\section{Data Sharing Statement}

All data generated or analyzed during this study were included in this published article, and no further data will be shared.

\section{Ethics Statement}

The local ethics committee (the Second Xiangya Hospital of Central South University, Hunan, China) approved the study (Registration number: ChiCTR-POC-17,010,431), and all patients gave informed consent.

\section{Acknowledgments}

We want to thank all the patients and clinicians who contributed to this study, especially the research assistant - Guoguo Zhong.

\section{Author Contributions}

All authors made substantial contributions to conception and design, acquisition of data, or analysis and interpretation of data; took part in drafting the article or revising it critically for relevant intellectual content; gave final approval of the version to be published; and agree to be accountable for all aspects of the work.

\section{Funding}

This work was supported by the National Natural Science Foundation of China (grant number 81770046) and CSU Xiangya Mingyi grant (2013).

\section{Disclosure}

The authors report no conflicts of interest in this work.

\section{References}

1. Vogelmeier CF, Criner GJ, Martinez FJ, et al. Global strategy for the diagnosis, management, and prevention of chronic obstructive lung disease 2017 report. GOLD executive summary. Am J Respir Crit Care Med. 2017;195(5):557-582. doi:10.1164/rccm.201701-0218PP

2. World Health Organization. World Health Organization (WHO) website. 2019. Available from: http://www.who.int. Accessed October 14, 2019.

3. Kessler R, Partridge MR, Miravitlles M, et al. Symptom variability in patients with severe COPD: a pan-European cross-sectional study. Eur Respir J. 2011;37(2):264-272. doi:10.1183/09031936.00051110

4. Partridge MR, Karlsson N, Small IR, et al. Patient insight into the impact of chronic obstructive pulmonary disease in the morning: an internet survey. Curr Med Res Opin. 2009;25(8):2043-2048. doi:10.1185/03007990903103006
5. Judith JS, Qian C, Michelle M, et al. Impact and factors associated with nighttime and early morning symptoms among patients with chronic obstructive pulmonary disease. Int J Chron Obstruct Pulmon Dis. 2015;10:577-586. doi:10.2147/COPD.S76157

6. Miravitlles M, Worth H, Soler Cataluña JJ, et al. Observational study to characterise 24-hour COPD symptoms and their relationship with patient-reported outcomes: results from the ASSESS study. Respir Res. 2014;15(122):119-129. doi:10.1186/s12931-014-0122-1

7. Miravitlles M, Izquierdo JL, Esquinas C, et al. The variability of respiratory symptoms and associated factors in COPD. Respir Med. 2017;129:165-172. doi:10.1016/j.rmed.2017.06.017

8. Kuyucu T, Güçlü SZ, Saylan B, et al. A cross-sectional observational study to investigate daily symptom variability, effects of symptom on morning activities and therapeutic expectations of patients and physicians in COPD-SUNRISE study. Tuberk Toraks. 2011;59 (4):328-339. doi:10.5578/tt.3268

9. Soler-Cataluña JJ, Sauleda J, Valdés L, et al. Prevalence and perception of 24-hour symptom patterns in patients with stable chronic obstructive pulmonary disease in Spain. Arch Bronconeumol. 2016;52(6):308-315. doi:10.1016/j.arbres.2015.11.010

10. Roche N, Small M, Broomfield S, et al. Real world COPD: association of morning symptoms with clinical and patient reported outcomes. COPD. 2013;10(6):679-686. doi:10.3109/15412555.2013.844784

11. Tsiligianni I, Metting E, van der Molen T, et al. Morning and night symptoms in primary care COPD patients: a cross-sectional and longitudinal study. An UNLOCK study from the IPCRG. NPJ Prim Care Respir Med. 2016;26:16040. doi:10.1038/npjpcrm.2016.40

12. Gary G, Brooke C, Leidy NK, et al. Development of the chronic obstructive pulmonary disease morning symptom diary (COPD-MSD). Health Qual Life Outcomes. 2016;14(1):104-118. doi:10.1186/s12955-016-0506-7

13. Marin JM, Beeh KM, Clemens A, et al. Early bronchodilator action of glycopyrronium versus tiotropium in moderate-to-severe COPD patients: a cross-over blinded randomized study (symptoms and pulmonary function in the moRnING). Int $J$ Chron Obstruct Pulmon Dis. 2016;11:1425-1434. doi:10.2147/COPD.S106127

14. Partridge MR, Miravitlles M, Ståhl E, et al. Development and validation of the capacity of daily living during the morning questionnaire and the global chest symptoms questionnaire in COPD. Eur Respir J. 2010;36(1):96-104. doi:10.1183/09031936.00123709

15. Xiaoyun L, Wei C, Jiaxi D, et al. Reliability and validity of the chronic obstructive pulmonary disease morning symptom diary Chinese version. Chin J Tuberc Respir Dis. 2019;42(6):444-450.

16. Anzueto A, Leimer I, Kesten S. Impact of frequency of COPD exacerbations on pulmonary function, health status and clinical outcomes. Int J Chron Obstruct Pulmon Dis. 2009;4:245-251. doi:10.2147/copd.s4862

17. Miravitlles M, Ferrer M, Pont A, et al. Effect of exacerbations on quality of life in patients with chronic obstructive pulmonary disease: a 2 year follow up study. Thorax. 2004;59(5):387-395. doi:10.1136/ thx.2003.008730

18. Halpin DM, Decramer M, Celli B, et al. Exacerbation frequency and course of COPD. Int J Chron Obstruct Pulmon Dis. 2012;7:653-661. doi:10.2147/COPD.S34186

19. Soler-Cataluña JJ, Martínez-García MA, Román Sánchez P, et al. Severe acute exacerbations and mortality in patients with chronic obstructive pulmonary disease. Thorax. 2005;60(11):925-931. doi:10.1136/thx.2005.040527

20. Hoogendoorn M, Hoogenveen RT, Rutten-van Mölken MP, et al. Case fatality of COPD exacerbations: a meta-analysis and statistical modelling approach. Eur Respir J. 2011;37(3):508-515. doi:10.1183/ 09031936.00043710

21. Lu M, Wang X, Cai B, et al. Perception of circadian variation of symptoms in Chinese patients with chronic obstructive pulmonary disease. $J$ Thorac Dis. 2017;9(10):3888-3895. doi:10.21037/ jtd.2017.08.131 
22. Hurst JR, Vestbo J, Anzueto A, et al. Susceptibility to exacerbation in chronic obstructive pulmonary disease. $N$ Engl J Med. 2010;363 (12):1128-1138. doi:10.1056/NEJMoa0909883

23. Moy ML, Teylan M, Weston NA, et al. Daily step count predicts acute exacerbations in a US cohort with COPD. PLoS One. 2013;8 (4):e60400. doi:10.1371/journal.pone.0060400

24. Beeh KM, Glaab T, Stowasser S, et al. Characterisation of exacerbation risk and exacerbator phenotypes in the POET-COPD trial. Respir Res. 2013;14(1):116. doi:10.1186/1465-9921-14-116

25. Burgel PR, Nesme-Meyer P, Chanez P, et al. Cough and sputum production are associated with frequent exacerbations and hospitalizations in COPD subjects. Chest. 2009;135(4):975-982. doi:10.1378/ chest.08-2062

26. Niewoehner DE, Lokhnygina Y, Rice K, et al. Risk indexes for exacerbations and hospitalizations due to COPD. Chest. 2007;131 (1):20-28. doi:10.1378/chest.06-1316
27. Quint JK, Baghai-Ravary R, Donaldson GC, et al. Relationship between depression and exacerbations in COPD. Eur Respir J. 2008;32(1):53-60. doi:10.1183/09031936.00120107

28. Zhou ZJ, Zhou AY, Zhao YY, et al. A comparison of the assessment of health status between CCQ and CAT in a Chinese COPD clinical population: a cross-sectional analysis. Int J Chronic Obstr. 2018; Volume 13:1675-1682. doi:10.2147/COPD.S161225

29. van Buul Amanda R, Kasteleyn Marise J, Chavannes Niels H, et al. Physical activity in the morning and afternoon is lower in patients with chronic obstructive pulmonary disease with morning symptoms. Respir Res. 2018;19:49. doi:10.1186/s12931-018-0749-4

30. van Buul AR, Kasteleyn MJ, Chavannes $\mathrm{NH}$, et al. The association between objectively measured physical activity and morning symptoms in COPD. Int J Chron Obstruct Pulmon Dis. 2017;12:28 31-2840. doi:10.2147/COPD.S143387

\section{Publish your work in this journal}

The International Journal of COPD is an international, peer-reviewed journal of therapeutics and pharmacology focusing on concise rapid reporting of clinical studies and reviews in COPD. Special focus is given to the pathophysiological processes underlying the disease, intervention programs, patient focused education, and self management protocols. This journal is indexed on PubMed Central, MedLine and CAS. The manuscript management system is completely online and includes a very quick and fair peer-review system, which is all easy to use. Visit http://www.dovepress.com/testimonials.php to read real quotes from published authors. 